\title{
S-Duality in Gauge Theories as a Canonical Transformation
}

\author{
Y. Lozano, * \\ Joseph Henry Laboratories, \\ Princeton University, \\ Princeton, New Jersey 08544, USA
}

\begin{abstract}
We show that S-duality in four dimensional non-supersymmetric abelian gauge theories can be formulated as a canonical transformation in the phase space of the theory. This transformation is the usual interchange between electric and magnetic degrees of freedom. It is shown that in phase space the modular anomaly emerges as the result of integrating out the momenta degrees of freedom. The generalization to $d$ dimensional abelian gauge theories of $p$-forms is also considered. In the case of non-abelian gauge theories a careful analysis of the constraints implied by the canonical transformation shows that it does not relate Yang-Mills theories with inverted couplings. In fact the dual theory is shown to be of Freedman-Townsend's type, also with $\tilde{\tau}=-1 / \tau$, $\tau=\frac{\theta}{2 \pi}+\frac{4 \pi i}{g^{2}}$.
\end{abstract}

PUPT-1552

hep-th/9508021

August 1995

*yolanda@puhep1.princeton. edu 


\section{Introduction}

A lot of progress has been made in the last few years in the understanding of S-duality as a symmetry of four dimensional gauge theories. The conjecture of Montonen and Olive [1] that $N=4$ supersymmetric Yang-Mills theories were invariant under strong-weak coupling with the exchange of the gauge group by its dual was tested in [2], were it was shown that in fact the partition function transformed as a modular form. Some progress has been also made for $N=2$ and $N=1$ supersymmetric Yang-Mills theories [3], 田. However a path integral derivation of S-duality is in general still unknown. In [8] Witten showed that S-duality in four dimensional abelian gauge theories [5, 6, 7] can be implemented at the level of the path integral in a very similar way to T-duality in non-linear sigma models in String Theory 12]. The idea is to consider a global isometry of the Lagrangian which can be expressed as translations of a given coordinate (the adapted coordinate), gauge this isometry by introducing a fake gauge field and impose the constraint that the curvature tensor associated to this gauge field is zero so that the gauge field is non-propagating. Integrating the Lagrange multiplier and fixing the gauge field to zero the original theory is recovered and integrating the gauge field and fixing the adapted coordinate to zero the new dual theory is obtained. In the case of T-duality the initial variables are 0-forms and the global isometry that is gauged is $\theta \rightarrow \theta+\epsilon$ where $\theta$ is the adapted coordinate. In the case of abelian gauge theories the initial variables are 1 -forms and the isometry which is gauged is $A \rightarrow A+\epsilon$ where now the $\epsilon$ parameter is a 1-form. Then the gauge field which has to be introduced is a 2 -form and its field strength a 3-form. In 4 dimensions the Lagrange multiplier imposing that the field strength vanishes is a 1-form, like the original gauge field, and the dual theory is expressed also in terms of 1-forms. Also for this non-supersymmetric case the partition function transforms as a modular function with a modular weight proportional to the Euler characteristic and the signature of the manifold [8, 9].

Given the analogy with T-duality a canonical transformation must be beyond this path integral manipulation, since this is the case in T-duality [14, 15, 16]. In section 2 we present the explicit generating functional producing this transformation and show that it is the generalization of the functional in 2-dimensional non-linear sigma models to 4 dimensions and 1 -forms. Under this transformation electric and magnetic degrees of freedom get interchanged (with the minus relative sign) as shown in abelian lattice gauge theories in [6]. The canonical transformation approach is the simplest in order to obtain the dual theory, also in this case in which in the Hamiltonian formulation one has to be careful with the constraints. It is easy to show that both the initial and the dual theory are defined in the same subspace of the phase space after the canonical transformation is performed. We show that in phase space the partition functions of the initial and dual theories coincide and that only after integrating out the momenta degrees of freedom the modular anomaly [8, 9] appears.

The same canonical transformation applied to the non-abelian case seems to relate YangMills theories with inverted couplings. However a careful analysis of the constraints points out that this is not the case. The dual theory is in fact of Freedman-Townsend's type [10], i.e. it is expressed as a function of arbitrary 2-forms which are not derived from a vector potential. We show this in section 3 .

The results presented in section 2 can be easily generalized to the case of $d$ dimensional

abelian gauge theories of $p$-forms, as it is explained in section 4 . The modular anomaly in 
the transformation of the partition function is obtained. The implementation at the level of the path integral using a coset construction was presented in [11].

\section{The abelian case}

In this section we construct the explicit canonical transformation which produces the change

$$
\tau \rightarrow-1 / \tau
$$

with $\tau=\theta / 2 \pi+4 \pi i / g^{2}$, for $U(1)$ four dimensional euclidean gauge theories.

Let us consider the Lagrangian

$$
\begin{aligned}
L & =\frac{1}{8 \pi}\left(\frac{4 \pi}{g^{2}} F_{m n} F^{m n}+\frac{i \theta}{4 \pi} \epsilon_{m n p q} F^{m n} F^{p q}\right) \\
& =\frac{i}{8 \pi}\left(\bar{\tau} F_{m n}^{+} F^{+m n}-\tau F_{m n}^{-} F^{-m n}\right)
\end{aligned}
$$

where

$$
\begin{aligned}
& F_{m n}^{+}=\frac{1}{2}\left(F_{m n}+{ }^{*} F_{m n}\right)=\frac{1}{2}\left(F_{m n}+\frac{1}{2} \epsilon_{m n p q} F^{p q}\right), \\
& F_{m n}^{-}=\frac{1}{2}\left(F_{m n}-{ }^{*} F_{m n}\right)=\frac{1}{2}\left(F_{m n}-\frac{1}{2} \epsilon_{m n p q} F^{p q}\right)
\end{aligned}
$$

and $F_{m n}=\partial_{m} A_{n}-\partial_{n} A_{m}$. It was shown in 80 that the transformation (2. 1) could be derived at the level of the path integral by the usual Rocek and Verlinde's procedure |13] one follows to construct abelian T-duals of two dimensional sigma models in String Theory. In this case given a global abelian continuous isometry of the sigma model one can turn it local by introducing a fake gauge field in the Lagrangian by minimal coupling and imposing the constraint that this gauge field is non-dynamical. Solving this constraint and fixing the gauge field to be zero one recovers the original theory. If instead the gauge field is integrated and the gauge is fixed in the original variables a sigma model written in terms of the Lagrange multiplier introduced to impose the constraint is obtained. This is the dual sigma model. In [8] the same construction is applied to obtain the dual of the four dimensional abelian gauge theory. The global continuous abelian isometry in this theory is

$$
A \rightarrow A+\epsilon
$$

where now the isometry parameter is a 1-form. This global isometry can be gauged by introducing a gauge field $G, 2$-form, which is imposed to be non-dynamical with the term

$$
\int_{M} d^{4} x \tilde{A} d G
$$

where the Lagrange multiplier $\tilde{A}$ is a 1 -form. Integrating $\tilde{A}$ the constraint $d G=0$ is obtained, ie. $G$ pure gauge, and we can recover (2.2) by either fixing $A=0$ or $G=0$. On the other hand by integrating out $G$ and then fixing $A=0$ the following Lagrangian is gotten:

$$
\tilde{L}=\frac{i}{8 \pi}\left(-\frac{1}{\bar{\tau}} \tilde{F}_{m n}^{+} \tilde{F}^{+m n}+\frac{1}{\tau} \tilde{F}_{m n}^{-} \tilde{F}^{-m n}\right)
$$


with $\tilde{F}^{ \pm}$the self- and antiself-dual components of $\tilde{F}_{m n} \equiv \partial_{m} \tilde{A}_{n}-\partial_{n} \tilde{A}_{m}$. This is the S-dual of the initial electromagnetic theory since in the particular case $\theta=0$ it corresponds to the inversion of the coupling constant $g$.

In this procedure we have made an integration by parts in the Lagrange multipliers term and neglected a total derivativet. However this total derivative contains some information, in particular it implies that the initial and dual Lagrangians are equal up to a total time derivative, exactly what happens when two theories are related by a canonical transformation. To be more precise, the generating functional of a canonical transformation from $\left\{q^{i}, p_{i}\right\}$ to $\left\{Q^{i}, P_{i}\right\}$ is such that

$$
p_{i} \dot{q}^{i}-H\left(q^{i}, p_{i}\right)=P_{i} \dot{Q}^{i}-\tilde{H}\left(Q^{i}, P_{i}\right)+\frac{d \mathcal{F}}{d t}
$$

If $\mathcal{F}$ is a type I generating functional (depending only on coordinates) $H=\tilde{H}$ if and only iff

$$
\begin{aligned}
& \frac{\partial \mathcal{F}}{\partial q^{i}}=p_{i} \\
& \frac{\partial \mathcal{F}}{\partial Q^{i}}=-P_{i}
\end{aligned}
$$

Under duality

$$
\tilde{L}(\tilde{A})=L(A)+d \tilde{A} \wedge d A
$$

which impliest

$$
\epsilon^{m n p q}\left(\partial_{m} \tilde{A}_{n}-\partial_{n} \tilde{A}_{m}\right)\left(\partial_{p} A_{q}-\partial_{q} A_{p}\right)=-\left(\frac{\delta \mathcal{F}}{\delta \tilde{A}_{m}} \dot{\tilde{A}}_{m}+\frac{\delta \mathcal{F}}{\delta A_{m}} \dot{A}_{m}\right)
$$

This produces the canonical transformation

$$
\begin{array}{ll}
\Pi^{\alpha}=\frac{\delta \mathcal{F}}{\delta A_{\alpha}}=-4^{*} \tilde{F}^{0 \alpha}, & \Pi^{0}=0, \\
\tilde{\Pi}^{\alpha}=-\frac{\delta \mathcal{F}}{\delta \tilde{A}_{\alpha}}=4^{*} F^{0 \alpha}, & \tilde{\Pi}^{0}=0
\end{array}
$$

plus a constraint

$$
\Pi^{\alpha} \partial_{\alpha} A_{0}=\tilde{\Pi}^{\alpha} \partial_{\alpha} \tilde{A}_{0},
$$

where greek indices run over spatial coordinates.

The generating functional producing this canonical transformation is

$$
\mathcal{F}=-2 \int_{M, \text { tfixed }} d^{3} x\left(\tilde{A}_{\alpha}{ }^{*} F^{0 \alpha}+A_{\alpha}{ }^{*} \tilde{F}^{0 \alpha}\right)=-\frac{1}{2} \int_{M} d^{4} x \tilde{F} \wedge F .
$$

\footnotetext{
${ }^{1}$ This term is seen in the gauge $A=0$.

${ }^{2}$ We assume $\mathcal{F}$ does not depend explicitly on time.

${ }^{3}$ We have dropped the global $i / 8 \pi$ factor. It will then appear when exponentiating these quantities.

${ }^{4}$ Our convention for the product of forms is: $\tilde{F} \wedge F=\epsilon^{m n p q} \tilde{F}_{m n} F_{p q}$.
} 
This is the result one would expect a priori from what is known in two-dimensional sigmamodels, where the generating functional is given in terms of the adapted coordinate to the isometry $\theta$ and the Lagrange multiplier $\tilde{\theta}$ by [14, 15]

$$
\mathcal{F}=-\frac{1}{2} \int_{M_{2}} d \tilde{\theta} \wedge d \theta
$$

The Hamiltonian associated to (2.2) is given by:

$$
H=\frac{1}{4(\bar{\tau}-\tau)} \Pi_{\alpha} \Pi^{\alpha}+\partial_{\alpha} A_{0} \Pi^{\alpha}-\frac{\bar{\tau}+\tau}{\bar{\tau}-\tau} \Pi_{\alpha}{ }^{*} F^{0 \alpha}+\frac{4 \bar{\tau} \tau}{\bar{\tau}-\tau}{ }^{*} F^{0 \alpha *} F_{0 \alpha}
$$

plus the constraints

$$
\Pi_{0}=0, \quad \partial_{\alpha} \Pi^{\alpha}=0,
$$

where

$$
\Pi^{\alpha}=4 \bar{\tau} F^{+0 \alpha}-4 \tau F^{-0 \alpha} .
$$

$\Pi_{0}$ is a primary constraint and $\partial_{\alpha} \Pi^{\alpha}=0$ is the secondary constraint emerging from the equation of motion for $\Pi_{0}$. They imply that the theory is defined in the reduced phase space given by $\Pi_{0}=0, \partial_{\alpha} \Pi^{\alpha}=0$. These constraints are also satisfied in the dual theory, since they are obtained directly from the canonical transformation. Then the dual theory is defined in the same reduced phase space than the original one. The relation (2.12) is trivial in this subspace. However we need to consider it in order to recover the dual Lagrangian from the canonically transformed Hamiltonian, since for that we need the naive Hamiltonian without taking into account the constraints. Our purpose is to show that the canonically transformed Lagrangian is the dual Lagrangian and for that we do not need to study in detail the way the theory gets defined in the Hamiltonian formalism [17], it is enough to show that both the initial and dual theories are defined in the same reduced phase space.

The canonically transformed Hamiltonian reads:

$$
\tilde{H}=\frac{1}{4} \frac{\bar{\tau} \tau}{\bar{\tau}-\tau} \tilde{\Pi}_{\alpha} \tilde{\Pi}^{\alpha}+\partial_{\alpha} \tilde{A}_{0} \tilde{\Pi}^{\alpha}+\frac{\bar{\tau}+\tau}{\bar{\tau}-\tau} \tilde{\Pi}_{\alpha}{ }^{*} \tilde{F}^{0 \alpha}+\frac{4}{\bar{\tau}-\tau}{ }^{*} \tilde{F}_{0 \alpha}{ }^{*} \tilde{F}^{0 \alpha}
$$

The corresponding Lagrangian is given by the dual Lagrangian (2.6). Recall that (2. 11):

$$
\begin{aligned}
& \Pi^{\alpha}=-4^{*} \tilde{F}^{0 \alpha}, \\
& \tilde{\Pi}^{\alpha}=4^{*} F^{0 \alpha}
\end{aligned}
$$

corresponds to the usual interchange between electric and magnetic degrees of freedom when there is no $\theta$-term.

Some useful information can be obtained within this approach. The generating functional (2. 13) is linear in both the original and dual variables. Then the following relation holds:

$$
H e^{\frac{i \mathcal{F}}{8 \pi}}=\tilde{H} e^{\frac{i \mathcal{F}}{8 \pi}}
$$

which implies:

$$
\psi_{k}[\tilde{A}]=N(k) \int \mathcal{D} A\left(x^{\alpha}\right) e^{\frac{i}{8 \pi} \mathcal{F}\left[\tilde{A}, A\left(x^{\alpha}\right)\right]} \phi_{k}\left[A\left(x^{\alpha}\right)\right]
$$


with $\phi_{k}[A]$ and $\psi_{k}[\tilde{A}]$ eigenfunctions of the initial and dual Hamiltonians respectively with the same eigenvalue and $N(k)$ a normalization factor [18]. From this relation global properties can be easily worked out. The Dirac quantization condition:

$$
\int_{\Sigma} F=2 \pi n, \quad n \in Z
$$

for $\Sigma$ any closed two-surface in the manifold, implies for $\tilde{F}$ :

$$
\int_{\Sigma} \tilde{F}=2 \pi m, \quad m \in Z
$$

and $\tilde{F}$ must live in the dual lattice. Also from (2. 20) the transformation applies to any four dimensional manifold $M$ since $\phi_{k}[A]$ can be the result of integrating the theory in an arbitrary manifold with boundary.

We can obtain in phase space the modular anomaly emerging in the transformation of the partition function [8, 9]. The argument goes as follows. In phase space the partition function is given by:

$$
Z_{p s}=\int \mathcal{D} A_{\alpha} \mathcal{D} \Pi^{\alpha} e^{-\frac{i}{8 \pi} \int d^{4} x\left(\dot{A}_{\alpha} \Pi^{\alpha}-H\right)}
$$

Under (2.11)

$$
\mathcal{D} A_{\alpha} \mathcal{D} \Pi^{\alpha}=\mathcal{D} \tilde{A}_{\alpha} \mathcal{D} \tilde{\Pi}^{\alpha} .
$$

Then the dual phase space partition function is given by:

$$
\tilde{Z}_{p s}=\int \mathcal{D} \tilde{A}_{\alpha} \mathcal{D} \tilde{\Pi}^{\alpha} e^{-\frac{i}{8 \pi} \int d^{4} x\left(\dot{\tilde{A}}_{\alpha} \tilde{\Pi}^{\alpha}-\tilde{H}\right)}=Z_{p s}
$$

showing that in phase space the partition function is invariant under duality. Integration on momenta in (2.23) gives:

$$
Z_{p s}=\int \mathcal{D} A_{\alpha}(\operatorname{Im} \tau)^{B_{2} / 2} e^{-\int d^{4} x L}
$$

with $L$ given by (2.2). The factor $(\operatorname{Im} \tau)^{B_{2} / 2}$ in the measure is the regularized $(\operatorname{det} \operatorname{Im} \tau)^{1 / 2}$ coming from the gaussian integration over the momenta. $B_{2}$ is the dimension of the space of 2 -forms in the four dimensional manifold $M$ (regularized on a lattice) and emerges because the momenta are 2-forms.

The same calculation in the dual phase space partition function gives:

$$
\tilde{Z}_{p s}=\int \mathcal{D} \tilde{A}_{\alpha}\left(\operatorname{det}\left(\operatorname{Im}-\frac{1}{\tau}\right)\right)^{1 / 2} e^{-\int d^{4} x \tilde{L}}
$$

with $\tilde{L}$ given by (2.6). We regularize the factor

$$
\left(\operatorname{det}\left(\operatorname{Im}-\frac{1}{\tau}\right)\right)^{1 / 2}=(\operatorname{det}(\operatorname{Im} \tau /(\tau \bar{\tau})))^{1 / 2}
$$

\footnotetext{
${ }^{5}$ In order to have a well-defined partition function we have to fix the gauge symmetry. The following arguments are in this sense formal.
} 
by

$$
(\operatorname{Im} \tau)^{B_{2} / 2} \bar{\tau}^{-B_{2}^{+} / 2} \tau^{-B_{2}^{-} / 2}
$$

where $B_{2}^{+}$and $B_{2}^{-}$are respectively the dimensions of the spaces of self-dual and anti-self-dual 2 -forms. In configuration space the partition function is defined by [8]:

$$
Z=(\operatorname{Im} \tau)^{\left(B_{1}-B_{0}\right) / 2} \int \mathcal{D} A_{\alpha} e^{-S}=(\operatorname{Im} \tau)^{\left(B_{1}-B_{0}-B_{2}\right) / 2} Z_{p s}
$$

and in the dual model

$$
\tilde{Z}=\left(\frac{\operatorname{Im} \tau}{\tau \bar{\tau}}\right)^{\left(B_{1}-B_{0}\right) / 2} \int \mathcal{D} \tilde{A}_{\alpha} e^{-\tilde{S}}
$$

From $Z_{p s}=\tilde{Z}_{p s}$ we arrive to

$$
Z=\tau^{-(\chi-\sigma) / 4} \bar{\tau}^{-(\chi+\sigma) / 4} \tilde{Z}
$$

where $\chi=2\left(B_{0}-B_{1}\right)+B_{2}$ is the Euler number (the regularization is such that $B_{p}=B_{4-p}$ ) and $\sigma=B_{2}^{+}-B_{2}^{-}$is the signature of the manifold. This is the modular factor appearing in [8, 9]. In phase space the partition function is simply defined as the integration over coordinates and momenta and it transforms as a scalar with modular weight equal to zero. Is only when going to the configuration space that the integrations over the momenta produce some determinants which after being regularized yield the modular factor found in [8, 9]. A very similar argument should apply to the transformation of the dilaton in two-dimensional non-linear sigma-models.

\section{The non-abelian case}

The canonical transformation approach can be generalized to the case of non-abelian gauge theories with compact group $G$. The initial Lagrangian is given by:

$$
\begin{aligned}
L & =\frac{1}{8 \pi}\left(\frac{4 \pi}{g^{2}} F_{m n}^{(a)} F^{(a) m n}+\frac{i \theta}{4 \pi} \epsilon^{m n p q} F_{m n}^{(a)} F_{p q}^{(a)}\right) \\
& =\frac{i}{8 \pi}\left(\bar{\tau} F_{m n}^{(a)+} F^{(a)+m n}-\tau F_{m n}^{(a)-} F^{(a)-m n}\right)
\end{aligned}
$$

where $F=d A-A \wedge A$ and we have chosen $\operatorname{Tr}\left(T^{a} T^{b}\right)=\delta^{a b}\left(T^{a}\right.$ are the generators of the Lie algebra). The conjugate momenta and the Hamiltonian are:

$$
\begin{gathered}
\Pi^{a \alpha}=2(\bar{\tau}-\tau) F^{(a) 0 \alpha}+2(\bar{\tau}+\tau)^{*} F^{(a) 0 \alpha} \\
\Pi^{a 0}=0 \\
H=\frac{1}{4} \frac{1}{\bar{\tau}-\tau} \prod_{\alpha}^{a} \Pi^{a \alpha}+\left(\partial_{\alpha} A_{0}^{a}+f_{a b c} A_{0}^{b} A_{\alpha}^{c}\right) \Pi^{a \alpha}-\frac{\bar{\tau}+\tau}{\bar{\tau}-\tau} \prod^{a \alpha *} F_{0 \alpha}^{(a)}+\frac{4 \bar{\tau} \tau}{\bar{\tau}-\tau}{ }^{*} F_{0 \alpha}^{(a) *} F^{(a) 0 \alpha},
\end{gathered}
$$

with $f_{a b c}$ the structure constants of the Lie algebra. The equations of motion of the primary constraints $\Pi^{a 0}=0$ imply:

$$
\partial_{\alpha} \Pi^{a \alpha}-f_{a b c} A_{\alpha}^{b} \Pi^{c \alpha}=0,
$$

so that we can ignore the second term in the Hamiltonian keeping in mind that the theory is defined in the reduced phase space given by the constraints. 
In the non-abelian case it proves more useful to use $\left\{{ }^{*} F_{0 \alpha}, \Pi^{\alpha}\right\}$ as the coordinates in phase space and look for a canonical transformation

$$
\left\{{ }^{*} F_{0 \alpha}, \Pi^{\alpha}\right\} \rightarrow\left\{{ }^{*} \tilde{F}_{0 \alpha}, \tilde{\Pi}^{\alpha}\right\} .
$$

Then in order to define correctly the phase space of the theory we have to introduce first order formalism for the initial Lagrangian. The idea is to introduce a Lagrangian $L[\tilde{F}, A]$, where now $\tilde{F}$ are arbitrary two-forms in the manifold, arranged to give $\tilde{F}=d A-A \wedge A$ from its equations of motion. Now the $\tilde{F}$ have no dynamical meaning since they have no time derivative, and the momenta are conjugate to the $A$-variables:

$$
\Pi^{a m}=\frac{\delta L[\tilde{F}, A]}{\delta \dot{A}_{m}^{a}} .
$$

It is easy to see that the following Lagrangian:

$$
L[\tilde{F}, A]=\frac{i}{8 \pi} \operatorname{Tr}\left(-\frac{1}{\bar{\tau}} \tilde{F}_{m n}^{+} \tilde{F}^{+m n}+\frac{1}{\tau} \tilde{F}_{m n}^{-} \tilde{F}^{-m n}-2\left(\tilde{F}_{m n}^{+} F^{+m n}-\tilde{F}_{m n}^{-} F^{-m n}\right)\right),
$$

with $F=d A-A \wedge A$, is such that (3.1) is obtained when solving the equations of motion for $\tilde{F}$. The canonical momenta are given by:

$$
\begin{aligned}
& \Pi^{a \alpha}=\frac{\delta L[\tilde{F}, A]}{\delta \dot{A}_{\alpha}^{a}}=-4^{*} \tilde{F}^{(a) 0 \alpha} \\
& \Pi^{a 0}=0
\end{aligned}
$$

and coincide with (3.2) when substituting the equations of motion. The Hamiltonian is also given by (3.3).

The canonical transformation

$$
\begin{aligned}
\Pi^{a \alpha} & =-4^{*} \tilde{F}^{(a) 0 \alpha} \\
\tilde{\Pi}^{a \alpha} & =4^{*} F^{(a) 0 \alpha},
\end{aligned}
$$

i.e. the usual interchange between electric and magnetic degrees of freedom, produces the following "dual" Hamiltonian:

$$
\tilde{H}=\frac{1}{4} \frac{\bar{\tau} \tau}{\bar{\tau}-\tau} \tilde{\Pi}^{a \alpha} \tilde{\Pi}_{\alpha}^{a}+\frac{\bar{\tau}+\tau}{\bar{\tau}-\tau} \tilde{\Pi}^{a \alpha *} \tilde{F}_{0 \alpha}^{(a)}+\frac{4}{\bar{\tau}-\tau}{ }^{*} \tilde{F}^{(a) 0 \alpha *} \tilde{F}_{0 \alpha}^{(a)},
$$

which is of the same form than the Hamiltonian of the initial theory with $\tilde{\tau}=-1 / \tau$. However one must be careful with the constraints. In particular the secondary constraints (3. 4) imply for the dual theory:

$$
\partial_{\alpha}{ }^{*} \tilde{F}^{(a) 0 \alpha}-f_{a b c} A_{\alpha}^{b}(\tilde{F})^{*} \tilde{F}^{(c) 0 \alpha}=0 .
$$

These equations are not satisfied by the Yang-Mills theory defined from $\tilde{F}$, so although (3. 10) would naively imply that the dual theory is a Yang-Mills theory with $\tilde{\tau}=-1 / \tau$, the analysis of the constraints shows that this is not the case. For the abelian theory the corresponding equation implies that $\tilde{F}$ is defined from a dual vector potential $\tilde{A}$, but the 
absence of a non-abelian analogue of Poincarè's lemma does not allow to conclude the same in the non-abelian case. Inversely if we would consider (3. 11) as an equation determining $A(\tilde{F})$ we would find incompatibility with $(3.9)$. We can then conclude that the usual interchange between electric and magnetic degrees of freedom does not relate Yang-Mills theories with inverted couplings.

Let us now obtain the "true" dual theory. The point is to realize that in (3. 7) we can integrate $A$ instead of $\tilde{F}$ and in this way a new theory is obtained. The equations of motion for $A$ are:

$$
\partial_{n}{ }^{*} \tilde{F}^{(a) m n}-f_{a b c} A_{n}^{b *} \tilde{F}^{(c) m n}=0,
$$

which imply:

$$
A_{m}^{a}=R_{m n}^{a b} \partial_{p}{ }^{*} \tilde{F}^{(b) n p},
$$

where $R$ is the inverse of $\operatorname{ad}^{*} \tilde{F}$ and it is a well defined matrix for arbitrary $\tilde{F}$ in four dimensions. Substituting in (3. 7) we get:

$$
\tilde{L}=\frac{i}{8 \pi}\left(-\frac{1}{\bar{\tau}} \tilde{F}_{m n}^{(a)+} \tilde{F}^{(a)+m n}+\frac{1}{\tau} \tilde{F}_{m n}^{(a)-} \tilde{F}^{(a)-m n}+2 R_{m n}^{a b}\left({ }^{*} \tilde{F}\right) \partial_{q}{ }^{*} \tilde{F}^{(a) q m} \partial_{p}{ }^{*} \tilde{F}^{(b) n p}\right),
$$

where $\tilde{F}$ are arbitrary two-forms in the manifold. This Lagrangian is of Freedman-Townsend's type [10], i.e. $\tilde{F}$ is the "fundamental" variable, not defined from a vector potential $\tilde{A}$, and it has been already shown to be dual to Yang Mills [19, 20, 21]. We should remark that in order to have a well defined dual theory a prescription must be given for the pole when $R$ becomes singular [21]. The Hamiltonian associated to (3.14) looks quite different from the Hamiltonian of Yang-Mills and it is not easy to see if they could be related by a canonical transformation. In any case such a transformation would have to map a phase space of two-forms $\left\{{ }^{*} F_{0 \alpha}, \Pi^{\alpha}\right\}$ into a phase space of two and one forms $\{\tilde{F}, \tilde{\Pi}\}$ (since the canonical momenta are conjugate to two forms), which means that if existing at all it would be quite different from an interchange between electric and magnetic degrees of freedom. Let us point out however that the first equation in (3. 9) (which maps two-forms on two-forms) produces the mapping from the constraint (3.4) of the initial theory to the equation of motion (3. 11) of the dual. This means that after all this interchange could have some physical meaning.

In this section we have been able to obtain the dual theory by manipulating the path integral in a similar way to the one known in the abelian case. One just needs to consider the intermediate Lagrangian $L[\tilde{F}, A]$ and integrate out either $\tilde{F}$ or $A$ to obtain the initial or the dual Lagrangians.

The group in which the dual variables live is the dual of the original group (the metric defined by its weight vectors is the inverse of the one in the original gauge group). In order to see this one needs to proceed more carefully in the previous derivation and take the original gauge fields in the fundamental representation of the gauge Lie algebra and the metric defined by the weight vectors $g_{a b} \equiv d_{a b}$. Following the steps explained above one arrives to a dual Lagrangian with metric $\tilde{g}_{a b}=\tilde{d}_{a b}$ where $\tilde{d}_{a b} d^{b c} \equiv \delta_{a}^{c}$.

\footnotetext{
${ }^{6}$ We have not written explicitly the determinant coming from the gaussian integration.
} 


\section{Generalization to p-forms abelian gauge theories}

The generalization to $p$-forms abelian gauge theories in $d$ dimensions is direct from what we have studied in section 27. We are going to consider the case $d=2(p+1)$ which is the one in which both the initial and dual theories are expressed as functions of $(p+1)$-forms 0 . The generalized S-duality transformation is implemented in the path integral by gauging the global isometry

$$
A \rightarrow A+\epsilon
$$

where now $A$ and the gauge parameter are $p$-forms. The total derivative term that gives information about the generating functional of the canonical transformation is $d \tilde{A} \wedge d A$, with $\tilde{A}$, the Lagrange multiplier, also a $p$-form.

It is immediate to show that the canonical transformation is generated by the type-I generating functionalf:

$$
F=-\frac{1}{(p+1) !} \int d^{d} x d \tilde{A} \wedge d A
$$

which produces:

$$
\begin{aligned}
\Pi^{\alpha_{1} \ldots \alpha_{p}} & =\frac{\delta F}{\delta A_{\alpha_{1} \ldots \alpha_{p}}}=-((p+1) !)^{2 *} \tilde{F}^{0 \alpha_{1} \ldots \alpha_{p}} \\
\tilde{\Pi}^{\alpha_{1} \ldots \alpha_{p}} & =-\frac{\delta F}{\delta \tilde{A}_{\alpha_{1} \ldots \alpha_{p}}}=((p+1) !)^{2 *} F^{0 \alpha_{1} \ldots \alpha_{p}}
\end{aligned}
$$

The same relation (2.20) for the wave functionals holds in this case since the generating functional is linear in the initial and dual variables. From it we can obtain global information about the dual variables. We can also obtain the modular weight appearing in the transformation of the partition function [11].

Let us consider first the case $p$ odd. $p+1$ is even and then the theory allows for a $\theta$-term. In phase space (we omit the $p$ indices):

$$
Z_{p s}=\int \mathcal{D} A \mathcal{D} \Pi e^{-\frac{i}{8 \pi} \int d^{d} x(\dot{A} \Pi-H)}=(\operatorname{Im} \tau)^{B_{p+1} / 2} \int \mathcal{D} A e^{-S},
$$

after regularizing the determinant coming from the gaussian integration on the momenta, $(p+1)$-forms in this case. The dual phase space partition function coincides with the initial one and it is given by:

$$
\tilde{Z}_{p s}=\int \mathcal{D} \tilde{\Pi} \mathcal{D} \tilde{A} e^{-\frac{i}{8 \pi} \int d^{d} x(\dot{\tilde{A}} \tilde{\Pi}-\tilde{H})}=(\operatorname{Im} \tau)^{B_{p+1} / 2} \tau^{-B_{p+1}^{-} / 2} \bar{\tau}^{-B_{p+1}^{+} / 2} \int \mathcal{D} \tilde{A} e^{-\tilde{S}}
$$

The configuration space partition function is:

$$
Z=(\operatorname{Im} \tau)^{N_{p} / 2} \int \mathcal{D} A e^{-S}
$$

\footnotetext{
${ }^{7}$ Dualization of spin-0 and spin-2 gravity-like theories has been studied in [22].

${ }^{8}$ In the arbitrary case the dual theory would depend on $(d-p-1)$ forms.

${ }^{9}$ Our conventions are: ${ }^{*} F^{i_{1} \ldots i_{p+1}}=\frac{1}{(p+1) !} \epsilon^{i_{1} \ldots i_{d}} F_{i_{p+2} \ldots i_{d}}$ and $\tilde{F} \wedge F=\epsilon^{i_{1} \ldots i_{d}} \tilde{F}_{i_{1} \ldots i_{p+1}} F_{i_{p+2} \ldots i_{d}}$.
} 
where we have followed the notation in [11], $N_{p}$ being the dimension of the space of $p$ forms after substracting all the gauge invariances (see [11] for the detailed analysis). In the dual model the partition function is the same with $\tau \rightarrow-1 / \tau$. Then we have:

$$
Z=\tau^{-\frac{\chi-\sigma}{4}} \bar{\tau}^{-\frac{\chi+\sigma}{4}} \tilde{Z}
$$

where $\chi=2(-1)^{p} N_{p}+(-1)^{p+1} B_{p+1}$ is the Euler number and $\sigma=B_{p+1}^{+}-B_{p+1}^{-}$the signature of the manifold.

In the case $p$ even a $\theta$-term does not exist. Similar arguments to the ones above yield:

$$
Z=\left(\frac{4 \pi}{g^{2}}\right)^{\chi / 2} \tilde{Z}
$$

All these results agree with the ones presented in [11].

\section{Conclusions}

We have seen that for non-supersymmetric abelian four dimensional gauge theories S-duality can be implemented as a canonical transformation in the phase space of the theory which is the usual interchange between electric and magnetic degrees of freedom. This transformation can be generalized to the case of non-abelian gauge theories, where it seems to yield a Yang-Mills theory with inverted couplings. However in this case the canonical transformation produces some constraints in the dual theory which in the absence of a non-abelian analogue of Poincarè's lemma do not imply that a dual vector potential exists. The dual theory is not a Yang-Mills theory but rather a Freedman-Townsend's type of theory [10] with inverted couplings. This is shown by defining an intermediate Lagrangian depending on the initial vector potential $A$ and the dual variables $\tilde{F}$ and from which the initial and dual Lagrangians are obtained by integrating $\tilde{F}$ or $A$ respectively. It is argued that if a canonical transformation relating the two theories exists it would be far different from an interchange between electric and magnetic degrees of freedom.

For the abelian case we have seen that in phase space the partition function is invariant under S-duality and it is only after integrating out the momenta degrees of freedom that a modular factor appears and the partition function in configuration space transforms as a modular function.

We have generalized the canonical transformation approach to $d$-dimensional abelian gauge theories defined with $p$ forms and obtained the corresponding modular weights appearing in the transformation of the partition function.

It could be very interesting to generalize the results presented in this paper to the case of supersymmetric gauge theories.

\section{Acknowledgements}

I would like to thank O. Alvarez and N. Mohammedi for useful discussions and especially J.L.F. Barbón for interesting remarks leading to the final form of this paper. A Fellowship from M.E.C. (Spain) is acknowledged for partial financial support. 


\section{References}

[1] C. Montonen and D. Olive, Phys. Lett. B72 (1977) 117.

[2] C. Vafa and E. Witten, Nucl. Phys. B431 (1994) 3.

[3] N. Seiberg and E. Witten, Nucl. Phys. B426 (1994) 19.

[4] N. Seiberg, Nucl. Phys. B435 (1995) 129.

[5] J. Cardy and E. Rabinovici, Nucl. Phys. B205 (1982) 1.

[6] J. Cardy, Nucl. Phys. B205 (1982) 17.

[7] A. Shapere and F. Wilczek, Nucl. Phys. B320 (1989) 669.

[8] E. Witten, On S-Duality in Abelian Gauge Theory, IASSNS-HEP-95-36, hepth/9505186.

[9] E. Verlinde, Global Aspects of Electric-Magnetic Duality, CERN-TH/95-146, hepth/9506011.

[10] D.Z. Freedman and P.K. Townsend, Nucl. Phys. B177 (1981) 282.

[11] J.L.F. Barbón, Nucl. Phys. B452 (1995) 313.

[12] A. Giveon, M. Porrati and E. Rabinovici, Phys. Rep. 244 (1994) 77; E. Alvarez, L. Alvarez-Gaumé and Y. Lozano, Nucl. Phys. B (Proc. Supp.) 41 (1995) 1.

[13] M. Roček and E. Verlinde, Nucl. Phys. B373 (1992) 630.

[14] A. Giveon, E. Rabinovici and G. Veneziano, Nucl. Phys. B322 (1989) 167; K.A. Meissner and G. Veneziano, Phys. Lett. B267 (1991) 33.

[15] E. Alvarez, L. Alvarez-Gaumé and Y. Lozano, Phys. Lett. B336 (1994) 183.

[16] Y. Lozano, Phys. Lett. B355 (1995) 165; O. Alvarez and C-H. Liu, Target-Space Duality between Simple Compact Lie Groups and Lie Algebras under the Hamiltonian Formalism: I. Remnants of Duality at the Classical Level, UMTG-184, hep-th/9503226; T. Curtright and C. Zachos, Phys. Rev. D49 (1994) 5408; D52 (1995) 573.

[17] P. Ramond, Field Theory: A Modern Primer, Addison Wesley.

[18] G.I. Ghandour, Phys. Rev. D35 (1987) 1289; T. Curtright, Differential Geometrical Methods in Theoretical Physics: Physics and Geometry, ed. L.L. Chau and W. Nahm, Plenum, New York, (1990) 279; and references therein.

[19] C. Hong-Mo, J. Faridani and T. Sheung Tsun, "A Non-abelian Yang-Mills Analogue of Classical Electromagnetic Duality", RAL-95-027, hep-th/9503106.

[20] N. Mohammedi, "Classical Duality in Gauge Theories", SHEP 95/23, hep-th/9507040. 
[21] O. Ganor and J. Sonnenschein, "The Dual Variables of Yang-Mills Theory and Local Gauge Invariant Variables", TAUP-2274-95, hep-th/9507036.

[22] T. Curtright and P. Freund, Nucl. Phys. B172 (1980) 413. 